\title{
Study on Constructing Digital 3D Model of Pumping Station Flow Channel
}

\author{
ZHAO Gang ${ }^{1, a}$, SHI Yin Tao ${ }^{2, b}$, Wang Mao Mei ${ }^{1, \mathrm{c}}$ \\ ${ }^{1}$ Jiangsu Provincial Hydraulic Research Institute, Nanjing, China, 210017 \\ ${ }^{2}$ Nanjing Tech University, Nanjing, China, 211800 \\ azgac@sina.com, byintaoshi@njtech.edu.cn, wangmaomei@163.com
}

Keywords: point clouds; registration; NURBS surface; 3D model;

Abstract. Pumping station flow channel is an important part of water pumping station, and plays an important role in the running process. The construction of the pumping station flow channel currently mainly depends on the pre-assembled wooden model, and the construction quality cannot be effectively assessed. With the help of the terrestrial 3D laser scanning technology, this paper mainly study on the construction of the digital 3D model of the pumping station flow channel by means of scanning, filtering, registration and modeling. The model not only apply on the analysis of the construction quality of the pumping station flow channel, but also on other simulation basis, such as simulation of section flow field, estimation of hydraulic loss values.

\section{Introduction}

Water pumping station flow channel have the features of typical surface structure, large-size templates and complex shape, and the construction quality control is difficult. In the course of construction, we usually use the prefabricated internal model as the core control links, and use total station for locating, Jack trimmer for precise positioning and template consolidation. At present, we measure the features section of the pumping station flow channel mainly by the means of precise level and high-precision total station, and simulate the gradual process in CAD software. Although the measurements of each characteristic cross section is of high precision, but the surface of the flow channel is surface gradient, the shape simulated by the several characteristics section have large difference with the actual shape that could not fully reflect the actual shape of the flow channel.

With the development of three-dimension scanning technology, point clouds have been widely used in three-dimensional digital simulation. Point clouds is the digitization result of physical surface information and constituted by a number of sampled points on the physical surface which describe a boundary representation of physical form. Point clouds have a number of advantages in digital simulation, such as simple structure, convenient access, fine detail description, and so on. This paper mainly discusses how to build a fine model of pumping station flow channel based on the collected point clouds.

\section{Acquiring Point-Clouds Data}

Point clouds collection consists of site reconnaissance, target layout and scan job.

\section{Site Reconnaissance}

Purpose of site reconnaissance is to design the whole scan scheme according to the characteristics of the pumping station flow channel, and choosing proper scan station location is main job. Under normal circumstances, the scan station settings should meet the following requirements.

I. Preset scan station should consider minimizing other shielding, and survey stations should have a certain degree of overlap regions.

II. According to the parameters of the selected scanner, the scan station and the target should keep the proper distance. 


\section{Target Layout}

After selected the scan station, we reasonably layout the target in accordance with the distribution of the scan station. Target arrangements usually change on the basis of the different operating environment, but follow certain common rules.

I. Two adjacent stations should contain at least three or more target location information.

II. Targets should be dispersed layout, and not placed on the same line or in the same vertical plane.

III. Under the licensing conditions, we should try to choose a spherical target.

\section{Scan Jobs}

After determining the scan scheme according to the site fact, scan job completed by using 3D laser scanner. In the scan job process, we adopted different scan resolutions for the pumping station flow channel and target, and guaranteed the adjacent sites coincide have at least 3 control targets. Firstly, we scan the whole scene by the low resolution; then, we scan the selected capture area by the normal resolution; Finally, we finely scan the target by the higher resolution. Since the coordinate system of the scanner unchanged from start to restart, we could not restart the scanner during the scan job in order to ensure that all the data are in the same coordinate system.

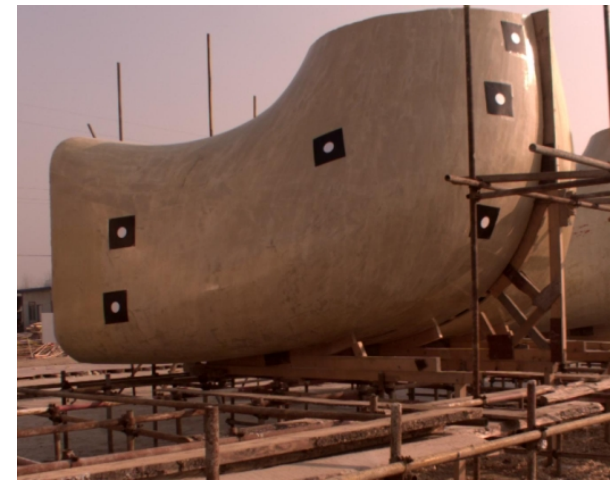

(a)Scan Sence

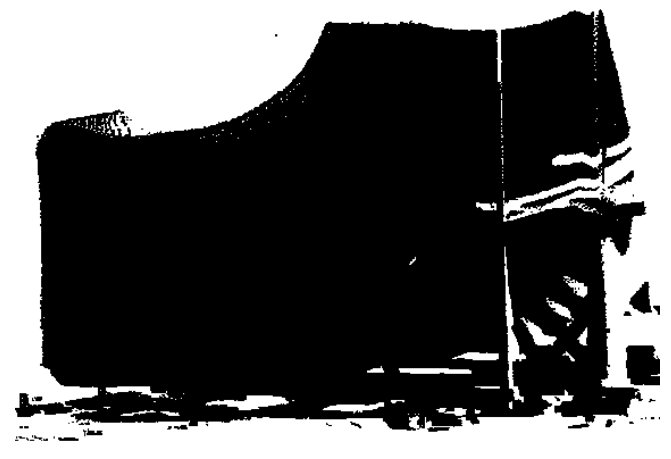

(b)Raw Point Clouds (371484 points)

Fig.1 Scan Job

\section{Constructing 3D fine model}

\section{Noise deletion}

In the process of scanning by the 3D laser scanner, some random unusual noises would occur when the laser pulse encounters the glass, metal, convex-concave surfaces and other similar goals. In particular situation the laser reflector is refracted back through the material specular surface, the measuring points will present a larger gross. At the same time, when scanning a particular target, we inevitably will scan objects around the target which results in unnecessary redundancy. Those points not only affect the overall data processing efficiency, and also affect the accuracy of the model, so they are usually referred to as noise. According to the spatial distribution of noises, they generally can be divided into four types of drift, isolation, redundant and mixed. For the first three categories, we usually can delete directly them by visual interactions, but the last kind of noises must be by means of points de-noising algorithm to remove.

For mixed types of noise, we choose the mean shift algorithm to remove it. Mean shift is the estimation of the density gradient in the space of two places, using statistics on the position of the distribution of points within a small area around. Mean shift move the points in space along the gradient direction until the gradient is zero. For the point cloud model $P \in R^{3}$, it includes spatial information $\boldsymbol{v}_{i}=\left(x_{i}, y_{i}, z_{i}\right)$ and normal vector $\boldsymbol{n}_{i}$, namely $\boldsymbol{p}_{i}=\left(\boldsymbol{v}_{i}, \boldsymbol{n}_{i}\right)$. Set the neighbor points of any point $\boldsymbol{p}_{\boldsymbol{i}}$ is $\left(\boldsymbol{p}_{i}\right)=\left\{\boldsymbol{q}_{i, 1}, \boldsymbol{q}_{i, 2}, \cdots, \boldsymbol{q}_{i, k}\right\}$, the mean shift vector can get by the Eq.1.

$$
M_{S}\left(\boldsymbol{p}_{i}\right)=\frac{\sum_{j=1}^{k} g\left(\left\|n_{i}-\boldsymbol{q}_{i, j}^{h}\right\|\right)\left(\boldsymbol{q}_{i, j}-M\left(p_{i}\right)\right)}{\sum_{j=1}^{k} g\left(\left\|n_{i}-\boldsymbol{q}_{i, j}^{h}\right\|\right)}
$$


In Eq.1, $g($.$) is generally Gaussian kernel functions, \boldsymbol{n}_{i}$ point normal vector, $\boldsymbol{q}_{i, j}^{h}$ is the neighbor information, $M_{S}\left(\boldsymbol{p}_{t}\right)$ is called mean shift points of the point $\boldsymbol{p}_{t}$. the initial value of $M\left(\boldsymbol{p}_{t}\right)$ is $\boldsymbol{p}_{t}, M_{S}\left(\boldsymbol{p}_{t}\right)$ is the mean shift vector of $M\left(\boldsymbol{p}_{t}\right)$. Therefore, the process of mean shift is progressive movement from the vertex to the sample.

$$
M\left(\boldsymbol{p}_{t}\right)=M\left(\boldsymbol{p}_{t}\right)+M_{S}\left(\boldsymbol{p}_{t}\right)
$$

From the iterative process, each point will converge to a stable point, known as the model point. In the repeated iteration process of mean shift, each point end finding the model point. When implemented in mean shift, we usually set the convergence condition of iteration process that $M_{S}\left(\boldsymbol{p}_{i}\right)$ is less than a certain positive value $\varepsilon$. Through the iterative process of Mean shift, each sampling point will be moved to one of its most likely position on the point set surfaces, which completed noise reduction of a point clouds model.

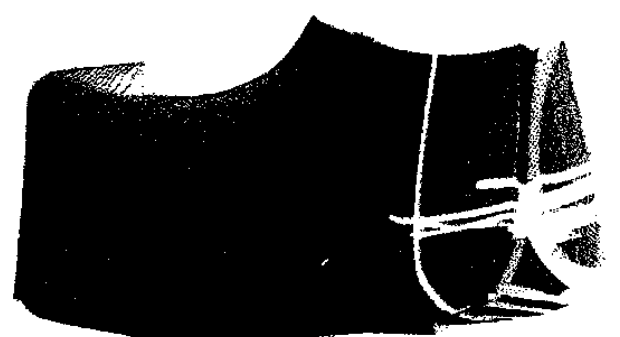

(a)After manual deletion(253218 points)

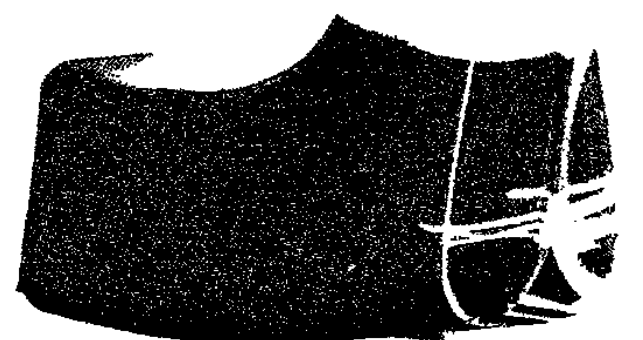

(b)After algorithm deletion (209843 points)

Fig.2 De-noise of Point Clouds

\section{Multi-site registration}

As the constraints of the external environment and the scan field of the 3D laser scanning system, the scanner can only gain part data of the pumping station flow channel surface in a single perspective. To build a pumping station flow channel model, we need to scan them in multiple perspectives, and converts the data from different perspective to the same coordinate's registration and fusion to obtain its complete geometric models, that is the site registration issues of the multi-site point clouds.

For the automatic registration of multi-site point clouds, we usually used the improved ICP (Iterative Closest Point) algorithm. The basic thought of the algorithm is: given target points set $p$ which need coordinates transform and reference points set $\mathrm{Q}$, in order to makes $\mathrm{p}$ and $\mathrm{Q}$ match, we firstly search each point in set $\mathrm{P}$ for the matched point in set $\mathrm{Q}$ that their distance are minimum and establish the mapping relationship between points, then calculate an optimal coordinates transform $\mathrm{M}$ by the least squares techniques, and makes $\mathrm{P}=\mathrm{M}(\mathrm{p})$, for Diego generation solution until precision meet the set one, eventual coordinates transform is the synthesis of each transform.

The steps of ICP algorithm are follows.

I. Assumed point clouds set $P$ and $\mathrm{Q}$ have overlapping region $\Omega$ and the position of any point in region $\Omega$ is respectively $p_{i}$ and $q_{j}$, we must find at least three pairs of nearest-neighbors $\left(p_{i}, q_{j}\right)$ for the registration of between set $\mathrm{P}$ and set $\mathrm{Q}$.

II. Calculate the distance from each point PI in $\mathrm{P}$ to every point in $\Omega$, and select the point pairs of the shortest distance as corresponding points. There is a shortest distance from every point in $\mathrm{P}$ to every point in $\mathrm{Q}$ among the overlap area. If there are i points in $\mathrm{P}$, there are i such shortest distance and i point pairs (pi, qi) of shortest distance in point set $\Omega$ of set $\mathrm{P}$ and $\mathrm{Q}$.

III. Calculate the transformation parameter between the set P and Q with the help of the selected point pairs $\left(p_{i}, q_{i}\right)$.

IV. Circularly carry out step II and III to get more accurate transformation parameters until meet the accuracy requirements.

V. Transform the point clouds $\mathrm{P}$ and $\mathrm{Q}$ with the calculated transformation parameters and complete the registration. 


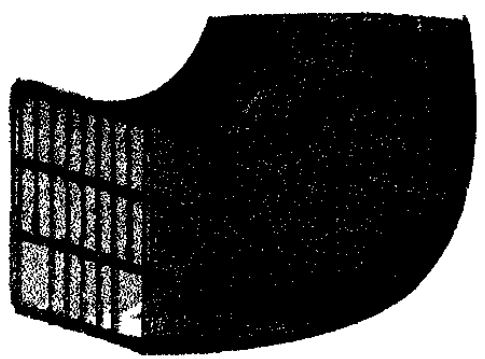

\section{Constructing NURBS Surface Model}

Fig.3 Registered Point Clouds

NURBS surface model have the characteristics of stable spatial geometry, local support and smooth continuity, and is suited for constructing surface model of pumping station flow channel. For a solid model with complex shape, fitting the overall surface by NURBS is difficult because the point clouds data itself is scattered and cannot directly form a rectangular array. So we divide the point clouds into different areas based on octree spatial segmentation, and fit the local surface by different region.

We construct a rectangular area of NURBS surfaces based on leaf nodes of octree, and approach to build the rest of the control points by the topological relationships between data points constructed by the characteristic variables. Thus, we divide the point clouds data into a series of grids, and then reconstruct the mesh surface of rectangular region on the basis of the grids.

In order to establish surfaces in accordance with point clouds, we require spatial division for the point clouds. Let the spatial index value of the cube where point $\mathrm{P}(\mathrm{x}, \mathrm{y}, \mathrm{z})$ locate be $\mathrm{P}(\mathrm{u}, \mathrm{v}, \mathrm{w})$, $\mathrm{p}=\mathrm{p}_{\mathrm{n}-1} \cdots \mathrm{p}_{\mathrm{i}} \cdots \mathrm{p}_{1} \mathrm{p}_{0}$ be the octal code of the nodes corresponding to the sub-cube. Given the codes of Octree node is $\mathrm{p}$, we can get the spatial index value $P(\mathrm{u}, \mathrm{v}, \mathrm{w})$ of the cubes the point locate by the Eq.3.

$$
\left\{\begin{array}{l}
\mathrm{u}=\sum_{\mathrm{i}=1}^{\mathrm{n}-1}\left(\mathrm{p}_{\mathrm{i}} \% 2\right) \times 2^{\mathrm{i}} \\
\mathrm{v}=\sum_{\mathrm{i}=1}^{\mathrm{n}-1}\left(\left(\mathrm{p}_{\mathrm{i}} / 2\right) \% 2\right) \times 2^{\mathrm{i}} \\
\mathrm{w}=\sum_{\mathrm{i}=1}^{\mathrm{n}-1}\left(\left(\mathrm{p}_{\mathrm{i}} / 4\right) \% 2\right) \times 2^{\mathrm{t}}
\end{array}\right.
$$

According to Eq.3, we can determine the spatial coordinates of the octree leaf nodes. We regard the leaf nodes as the four control points of the reconstructed surface and gain other control points by interpolation with spline basis functions.

If the division of the point clouds octree is enough fine and the shape of the local rectangular area surface is not complicated, we can adopt cubic uniform B-spline approximation to fit the rectangular region for reduction of the surface fitting. After generated the NURBS surface, four surfaces constitute leaf node of quad-tree, and form a surface recursive structure with pointers. Every four little quadrilateral grids corresponding to the control grid have one common ground that is the interaction points of the four grids. Corresponding to each of the common interaction points, we establish links with the quad-tree nodes to create a new grid. After each merge, old mesh would be replaced by the new one. Through that recursive subdivision, we eventually fit the grid into a composite surface. The surface quad-tree generates by means of post-order-traversing, and the root pointers are regarded as identification. Multiple interrelated NURBS surfaces of the solid model are interconnected, and the entire surface would be progressively redrawn via post-order-traversing quad-tree in the process of visualization.

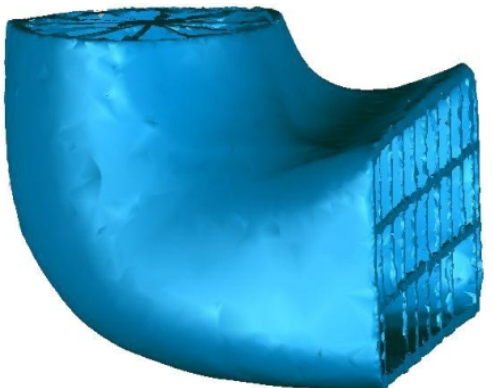

Fig.4 Constructed NUBRS Model 


\section{Conclusion}

Terrestrial laser scanning technology is widely used in various fields, such as digital city and 3D architectural models, relic's protection, route selection of municipal roads and power line, topographic surveys, etc. This article will apply that on the digital 3D modeling of pumping station flow channel, and the constructed model not only can be used to analyze the construction deviation of between the actually constructed flow channel and the design one, but also as a base for other simulation, such as the simulation of cross section flow field and estimation of hydraulic loss values.

\section{Acknowledgements}

This paper is funded by the Jiangsu Provincial Surveying and Geo-information research project (JSCHKY201511).

\section{Reference}

[1] Boissonnat J. Geometric structures for three-dimensional shape representation. ACM Transaction on Graphics, 1984, vol.4(9), p.266-286.

[2] Varady T, Martin R. Reverse engineering of geometric models-an introduction. Computer-Aided Design, 1997, Vol.29(6), p.255-268.

[3] Stamminger M., Drettakis G. Interactive sampling and rendering for complex and procedural geometry. Proceedings of the 12th Euro graphics Workshop on Rendering, 2001, p.76-89.

[4] Yamany S., Farag A. Surface signatures: an orientation independent free-form surface representation scheme for the purpose of objects registration and matching. IEEE Transactions on Pattern Analysis and Machine Intelligence, 2002, Vol.24 (8), p.1105-1120.

[5] Lee H., Woo T. Point Data Reduction Using 3D Meshs. The International Journal of Advanced Manufacturing Technology, 2001,Vol.18, p.201-210.

[6] Dey T., Giesen J. Detecting under sampling in surface reconstruction. Proceedings of the 17th ACM Symposium Computational Geometry, 2001, p.257-263.

[7] Levin D. Mesh-independent surface interpolation. Geometric Modeling for Scientific Visualization 2003, p.37-39. 